\title{
Gravitons in a Box
}

\author{
Sougato Bose, ${ }^{1}$ Anupam Mazumdar, ${ }^{2}$ and Marko Toros ${ }^{3}$ \\ ${ }^{1}$ Department of Physics and Astronomy, University College London, Gower Street, WC1E 6BT London, UK \\ ${ }^{2}$ Van Swinderen Institute, University of Groningen, 9747 AG, The Netherlands \\ ${ }^{3}$ School of Physics and Astronomy, University of Glasgow, Glasgow, G12 8QQ, UK
}

\begin{abstract}
Gravity and matter are universally coupled, and this unique universality provides us with an intriguing way to quantifying quantum aspects of space-time in terms of the number of gravitons within a given box. In particular, we will provide a limit on the the number of gravitons if we trace out the matter degrees of freedom. We will obtain the universal bound on the number of gravitons, which would be given by $N_{g} \approx\left(m / M_{p}\right)^{2}$. Since the number of gravitons also signifies the number of bosonic states they occupy, the number of gravitons will indirectly constrain the system's gravitational entropy. We will show that it saturates Bekenstein bound on the gravitational Arealaw of entropy. Based on these observations, we will ascertain that the gravitons permeating in the observable Universe always $N_{g} \gg 1$.
\end{abstract}

Introduction: Within the general theory of relativity, the dynamics of space-time is intertwined by both matter and the gravitational degrees of freedom. If both matter and the gravitational degrees of freedom are quantum in nature, then their interplay will pave an important role in any quantum theory of gravity [1]. However, there is no experimental proof yet to show that gravity is quantum in nature.

Like other forces of nature, the spin-2 graviton is thought to be the carrier of the gravitational interaction, and it is responsible for the gravitational attraction between two massive bodies. A graviton can be canonically quantised around a weak curvature background [2], and we can attribute both on-shell and off-shell degrees of freedom to the massless graviton. The former is responsible for describing independent dynamical modes, while the latter describes how the force is being mediated between two massive bodies. One of the interesting features of the gravitational interaction is that it is universal, and it is governed by Newton's constant $G \sim 1 / M_{p}^{2}$, where $M_{p} \sim 1.2 \times 10^{19} \mathrm{GeV}$.

Niels Bohr once argued, double-slit experiment with an electron, that the photon which mediates electromagnetic interaction ought to be quantum if the electron is a bonafide quantum entity, see [3]. The purported weakness of the universal gravitational interaction with the matter precluded a similar argument favouring the quantum nature of a graviton. A similar weakness in the gravitational interaction was poignantly used by Dyson to point out that it would be extremely challenging to detect gravitons [4]. Although, very tiny but a quantum graviton interaction with matter can leave indelible mark in classical/quantum systems [5-10].

In fact, just quantum mechanics, along with the special theory of relativity, suggests that a graviton exchange between two quantum superposed masses can provide a bonafide quantum feature if the two quantum systems are allowed to interact solely gravitationally. In fact, the two quantum systems can be entangled by a graviton exchange in a Feynman diagram, which can be tested via a scheme known as the quantum gravity induced entanglement of masses (QGEM) 9, 11]. In any given experiment, the number of gravitons, either on-shell or off-shell, can leave a detectable mark on observations. In most typical cases, for general open systems, the number of quanta is also indicative of whether a quantum system can be approximated by the mean field or a statistical ensemble 1 .

This paper aims to point out that there is a universal bound in any quantum interaction of a graviton with the matter degrees of freedom. For the time being, we neglect any other interaction besides gravity. This universal behaviour can be studied by tracing out the matter degrees of freedom - we will show that the occupation number of gravitons is always proportional to the Area-law of such a gravitational system. Intriguingly, such a behaviour can be thought to be universal from the point of view of Bekenstein's bound on a gravitational entropy [15] (while it has been argued that physically realistic local quantum Hamiltonians in the ground state follow the Area-law of entanglement entropy [16, 17]). Since in a thermodynamic system, the entropy is proportional to the number of gravitons, and entropy is saturated by the Area-law, no wonder by tracing out all the matter degrees of freedom, we obtain that the Area-law always bounds the occupation number of gravitons.

Graviton coupling to matter: To set up our computation, let us assume that we work in a world line of a particle in a Fermi-normal coordinate, and without loss of generality, let us assume that the particle motion is in the $x$-axis. Let us consider an ideal matter following a geodesic trajectory, $x^{\mu}$ (the situation of an observer following a generic time-like curve can be analysed similarly without affecting the final results). We will start

\footnotetext{
${ }^{1}$ Number or density plays an important role, there are roughly 400 microwave photons per cubic centimetre, and its fluctuations have been discovered in terms of two-point temperature correlations by the latest Planck experiments [12]. However, we still do not know whether these fluctuations are classical or quantum [13, 14].
} 
from the general relativistic point-particle Lagrangian2:

$$
L=-m \sqrt{g_{\mu \nu} \dot{x}^{\mu} \dot{x}^{\nu}},
$$

where $m$ is the mass of the system, and $g_{\mu \nu}$ is the metric expressed in Fermi normal coordinates. In particular, we will write the metric as $g_{\mu \nu}=\eta_{\mu \nu}+h_{\mu \nu}, \eta_{\mu \nu}$ is the Minkowski metric, and $h_{\mu \nu}$ is the spacetime curvature perturbation near the geodesic up to order $\mathcal{O}\left(x^{2}\right)$.

Let us assume that the matter is moving slowly, the dominant contribution to the dynamics will be given by [20]:

$$
g_{00}=-\left(1+\ddot{h}_{11} x^{2}\right),
$$

where $\ddot{h}_{11}=2 R_{0101}$ is the "+" component of the gravitational waves usually discussed in the transverse-traceless (TT) coordinates, and $R$ is the Riemann tensor (here $\ddot{h}_{11} \equiv \ddot{h}_{11}(t, 0)$ denotes only a number evaluated on the reference geodesic).

Now, let is use Eqs. (11) and (2), we can then readily find the interaction Lagrangian between graviton and matter degrees of freedom:

$$
L_{\mathrm{int}}=\frac{m}{4} \ddot{h}_{11} x^{2} .
$$

Let us now expand the gravitational fluctuation in terms of Fourier modes 3 , see Refs. [21]:

$$
h_{i j}(t, \boldsymbol{x})=\int d \boldsymbol{k} \sqrt{\frac{G \hbar}{\pi^{2} \omega_{k}}} g_{\boldsymbol{k}, \lambda} \mathrm{e}_{i j}^{\lambda}(\boldsymbol{n}) e^{-i\left(\omega_{k} t-\boldsymbol{k} \cdot \boldsymbol{x}\right)}+\text { H.c. },
$$

where $G$ is the Newton's constant, $\omega_{k}=k, k=\|\boldsymbol{k}\|$, $\boldsymbol{n}=\boldsymbol{k} /\|\boldsymbol{k}\|$, and $g_{\boldsymbol{k}, \lambda}$ is the annihilation operator. In Eq. (4) we also implicitly assume the summation over the polarization $4, \sum_{\lambda}$, where $\mathrm{e}_{j k}^{\lambda}$ denote the basis tensors for the two polarizations, $\lambda=1,2$. From Eq. (3) and (4) we however see that only $\mathrm{e}_{11}^{\lambda}(\boldsymbol{n})$ is relevant and we can write also the corresponding kinetic term for the massless graviton field to be:

$$
\begin{aligned}
H_{\text {grav }} & =\int d \boldsymbol{k} \hbar \omega_{k} g_{\boldsymbol{k}, \lambda}^{\dagger} g_{\boldsymbol{k}, \lambda} \\
& =\sum_{\lambda} \int d \boldsymbol{k} \frac{\hbar \omega_{k}}{4}\left[P_{\boldsymbol{k}, \lambda}^{2}+Y_{\boldsymbol{k}, \lambda}^{2}\right],
\end{aligned}
$$

where

$$
Y_{\boldsymbol{k}, \lambda}=g_{\boldsymbol{k}, \lambda}+g_{\boldsymbol{k}, \lambda}^{\dagger} \quad P_{\boldsymbol{k}, \lambda}=i\left(g_{\boldsymbol{k}, \lambda}^{\dagger}-g_{\boldsymbol{k}, \lambda}\right) .
$$

\footnotetext{
2 We assume $c=1$ here, and we work with $(-,+,+,+)$ signature, and $\mu=0,1,2,3$.

3 The notion of Fourier modes implicitly assumes that we are in an asymptotically flat space times, and free from curvature singularities.

4 The basis tensors satisfy the completeness relation: $\sum_{\lambda} \mathrm{e}_{i j}^{\lambda}(\boldsymbol{n}) \mathrm{e}_{k l}^{\lambda}(\boldsymbol{n})=P_{i k} P_{j l}+P_{i l} P_{j k}-P_{i j} P_{k l} \quad$ where $P_{i j} \equiv P_{i j}(\boldsymbol{n})=\delta_{i j}-\boldsymbol{n}_{i} \boldsymbol{n}_{j}$. For later convenience we write the integral: $\int d \boldsymbol{n} P_{11}(\boldsymbol{n}) P_{11}(\boldsymbol{n})=32 \pi / 15$.
}

The interaction Hamiltonian can be derived from the interaction Lagrangian in Eq. (3), and by using Eq. (4), we obtain [22]:

$$
H_{\mathrm{int}}=-m \sum_{\lambda} \int d \boldsymbol{k} e_{11}^{\lambda}(\boldsymbol{n}) \mathcal{C}_{\boldsymbol{k}} Y_{\boldsymbol{k}, \lambda} x^{2}
$$

where $x$ is the position operator of the particle, and

$$
\mathcal{C}_{k}^{\lambda}=\sqrt{\frac{G \hbar \omega_{k}^{3}}{16 \pi^{2}}}
$$

The interaction in Eq. (7) assumes long-wavelength gravitons such that the cutoff frequency $\bar{\omega}=2 \pi / l$. We note that by combining Eqs. (5) and (7), we find the relevant part of the gravitational Hamiltonian:

$$
\begin{aligned}
H= & \sum_{\lambda} \int d \boldsymbol{k} \frac{\hbar \omega_{k}}{4}\left[P_{\boldsymbol{k}, \lambda}^{2}+Y_{\boldsymbol{k}, \lambda}^{2}\right] \\
& -m \sum_{\lambda} \int d \boldsymbol{k} e_{11}^{\lambda}(\boldsymbol{n}) \mathcal{C}_{\boldsymbol{k}} Y_{\boldsymbol{k}, \lambda} x^{2} .
\end{aligned}
$$

The key points here are that we treat both matter and graviton on an equal footing, when it comes to quantum in nature, and we are dealing with a self-gravitating quantum system of mass $m$, whose interaction with graviton is determined by Eqs. (77).

Tracing out matter degrees of freedom: Suppose we consider the mean field approximation of the matter sector in Eq. (9), i.e. $x^{2} \rightarrow\left\langle x^{2}\right\rangle$, which is applicable in our case, because the gravitational coupling to matter degrees of freedom is very weak, suppressed by $G \sim 1 / M_{p}^{2}$. Furthermore, we are far away from any pathologies in space time, which allows us to work within the leading order contribution in $G \sim 1 / M_{p}^{2}$, it is a good assumption that the states of the graviton and the matter sector are factorizable. From Eq. (9) we thus find a displaced harmonic trap for the gravitational field:

$$
H=\sum_{\lambda} \int d \boldsymbol{k} \frac{\hbar \omega_{k}}{4}\left[P_{\boldsymbol{k}, \lambda}^{2}+\left(Y_{\boldsymbol{k}, \lambda}-\alpha_{\lambda, \boldsymbol{k}}\right)^{2}\right],
$$

where the center is given by $\alpha_{\lambda, \boldsymbol{k}} \equiv \frac{2 m}{\hbar \omega_{k}} e_{11}^{\lambda}(\boldsymbol{n}) \mathcal{C}_{\boldsymbol{k}}\left\langle x^{2}\right\rangle$. Now, let us suppose that the gravitational field mode $g_{\boldsymbol{k}, \lambda}$ is in a ground state, centred around $\alpha_{\boldsymbol{k}}^{\lambda}$, which can be described by a displaced coherent state,

$$
\left|\alpha_{\boldsymbol{k}}^{\lambda}\right\rangle=D\left(\alpha_{\boldsymbol{k}}^{\lambda}\right)|0\rangle=e^{\alpha_{\boldsymbol{k}}^{\lambda}\left[g_{\boldsymbol{k}, \lambda}^{\dagger}+g_{\boldsymbol{k}, \lambda}\right]}|0\rangle .
$$

By choosing the gravitational field to be in the ground state of the displaced harmonic trap we envisage that the matter system and the gravitational field have reached a steady-state - a different choice for the gravitational field state will not change significantly the final result as long as the state remains centered and confined around the same minimum. For such a displaced quantum state we can compute the expectation values $\langle\cdot\rangle-$ here we will 
be interested in the mean value and fluctuations of the number operator $N_{\boldsymbol{k}, \lambda} \equiv g_{\boldsymbol{k}, \lambda}^{\dagger} g_{\boldsymbol{k}, \lambda}$, where the gravitons follow the commutation relations: $\left[g_{\boldsymbol{k}, \lambda}, g_{\boldsymbol{k}^{\prime}, \lambda^{\prime}}^{\dagger}\right]=\delta(\boldsymbol{k}-$ $\left.\boldsymbol{k}^{\prime}\right) \delta_{\lambda, \lambda^{\prime}}$.

We find the occupation number of $g_{\boldsymbol{k}, \lambda}$ gravitons in the ground state to be:

$$
\left\langle N_{\boldsymbol{k}, \lambda}\right\rangle=\left|\alpha_{\boldsymbol{k}}^{\lambda}\right|^{2}=\left(\frac{2 m}{\hbar \omega_{k}} e_{11}^{\lambda}(\boldsymbol{n}) \mathcal{C}_{\boldsymbol{k}}\left\langle x^{2}\right\rangle\right)^{2} .
$$

The total number of gravitons can be then computed by summing and integrating over all the graviton modes:

$$
N_{g} \equiv \sum_{\lambda} \int d \boldsymbol{k}\left\langle N_{\boldsymbol{k}, \lambda}\right\rangle=\sum_{\lambda} \int d \boldsymbol{k}\left[\frac{2 m}{\hbar \omega_{k}} e_{11}^{\lambda}(\boldsymbol{n}) \mathcal{C}_{\boldsymbol{k}}\left\langle x^{2}\right\rangle\right]^{2} .
$$

By using Eq. (8) and footnote 4, we note that

$$
N_{g}=\int k^{2} d k \frac{4 m^{2}}{\hbar^{2} \omega_{k}^{2}} \int d \boldsymbol{n} \sum_{\lambda} e_{11}^{\lambda}(\boldsymbol{n}) e_{11}^{\lambda}(\boldsymbol{n}) \mathcal{C}_{\boldsymbol{k}}^{2}\left\langle x^{2}\right\rangle^{2},
$$

reduces to

$$
N_{g}=\frac{8 m^{2} G}{15 \pi \hbar}\left\langle x^{2}\right\rangle^{2} \int_{0}^{\bar{\omega}} d \omega_{k} \omega_{k}^{3}
$$

where $\bar{\omega}$ is the cutoff frequency for a box of side $l$.

Number of gravitons: We can perform the integration in Eq. (15), and by using $\bar{\omega}=2 \pi / l$, we find a factor $(2 \pi / l)^{4} / 4$. Furthermore, we note that $\left\langle x^{2}\right\rangle^{2} \leq l^{4} / 2^{4}$. We then find that the $l$ dependence goes away from the numerator and the denominator in Eq. (15), leaving us with a straightforward relationship, where the number of gravitons is simply given by the mass $m$, and Newton's constant, $G$ :

$$
N_{g} \leq \frac{4 \pi^{3}}{30} \frac{G m^{2}}{\hbar}=\frac{4 \pi^{2}}{30}\left(\frac{\text { Area }}{4 G \hbar}\right) .
$$

In the above, by area we mean the area corresponding to the Schwarzschild radius of mass $m$, given by $R_{s}=2 G m$, which is also the natural length-scale over which the above number of gravitons are confined. Same result, aside minor numerical factors, is obtained for a relativistic energy-momentum tensor. 5

Since the entropy of a black hole is proportional to the Area of the hole, the number of gravitons found above naturally scales as Bekenstein's entropy $N_{g} \sim S_{g}$, or

\footnotetext{
${ }^{5}$ Suppose we had taken an example of a relativistic energymomentum tensor $T_{\mu \nu}$, where the graviton-matter interaction would be dictated by the interaction $\sqrt{G} h_{\mu \nu} T^{\mu \nu}$, and we would then quantise the graviton in a transverse traceless (TT)-gauge, we would get a similar answer barring the numerical factors, $N_{g} \leq\left(32 \pi^{3} / 105\right)\left(G m^{2} / \hbar\right)$.
}

specifically, it is consistent with the bound set by Bekenstein, i.e. $N_{g} \sim S_{g}<2 \pi E R / \hbar$, where $E$ is total massenergy of the system, and $R$ is the characteristic radius, in the black hole case $E R \sim 2 G m^{2}[15]$. At this point, it has not avoided our attention that the gravitational entropy is indeed holographic [18, 19].

An intriguing feature of the above expression is that the number of gravitons signifies how we may be able to quantify the quantum nature of space-time itself. If we can squeeze the matter within the Schwarzschild radius, the number of gravitons saturate the black hole entropy. In GR, Schwarzschild radius is the only length scale that appears if we place a mass $m$ in an asymptotically flat space-time. In fact, from Eq. (16) we can roughly estimate the number of gravitons to be:

$$
N_{g} \approx\left(m / M_{p}\right)^{2} .
$$

For a solar mass blackhole $m \sim 10^{33} \mathrm{~g}$, and $M_{p} \sim 10^{-5} \mathrm{~g}$, $N_{g} \sim 10^{76}$. The large $N_{g} \geq 1$, sets a barrier for any massive quantum system, whose mass exceeds that of the gravitational mass $m \geq M_{p} \sim 10^{-5} \mathrm{~g}$. From Bohr's correspondence principle we may be able to treat such self-gravitating system approaching towards a classical limit.

The notion that large values of $N_{g}$ can be associated with classical behaviour is further reinforced by computing the quantum fluctuations,

$$
\Delta N_{g} \equiv \sqrt{\left\langle N^{2}\right\rangle-\langle N\rangle^{2}},
$$

of the total number operator $N \equiv \sum_{\lambda} \int d \boldsymbol{k} N_{\boldsymbol{k}, \lambda}$. We have already calculated $\langle N\rangle=N_{g}$ and using the commutation relations $\left[g_{\boldsymbol{k}, \lambda}, g_{\boldsymbol{k}^{\prime}, \lambda^{\prime}}^{\dagger}\right]=\delta\left(\boldsymbol{k}-\boldsymbol{k}^{\prime}\right) \delta_{\lambda, \lambda^{\prime}}$ we can show $\left\langle N^{2}\right\rangle=N_{g}+N_{g}^{2}$ (see for example 23]). Inserting the expectation values back in Eq. (18) we then find that the fluctuations grow only as $\Delta N_{g}=\sqrt{N_{g}}$. Hence we find that the relative quantum fluctuations follow as

$$
\Delta N_{g} / N_{g} \sim 1 / \sqrt{N_{g}}
$$

in line with the expectation that the system appears classical for large values of $N_{g} \gg 1$.

A curious reader would wonder if we were to trace the graviton degrees of freedom instead of matter degrees of freedom. In such a case, $Y_{\boldsymbol{k}, \lambda} \rightarrow\left\langle Y_{\boldsymbol{k}, \lambda}\right\rangle$ in Eq. (9), and we would be left with a simple harmonic oscillator potential. For such a system, the occupation number $N_{m}$ will not follow the Area-law. We can further ask whether the Area-law is a generic property for all massless fields or specific to the matter-graviton coupling. We recall that the quadratic nature of the coupling $\sim x^{2}$ in Eq. (3) was critical: we have shown $N_{g} \propto\left\langle x^{2}\right\rangle$ - where $\left\langle x^{2}\right\rangle$ is always non-zero - leading to the Area-law. In contrast, a linear coupling, e.g., $\sim x$, can always be cancelled by a change of the reference frame and should thus not play a role in the derivation of the Area-law. More generally one 
can expect the emergence of an Area law whenever the coupling gives rise to a length scale, while for other types of interactions (such as the electromagnetic) one is left without such interpretation.

Applications and features: Let us now provide another intriguing connection to what we have just found in Eqs. (16) and (17). In Refs. [24 27], see Ref. [28] for earlier discussions, a very interesting idea has been developed, a corpuscular nature of a black hole. A black hole is perceived to be a Bose-Einstein condensate of a large number of weakly interacting gravitons, where the authors have found exactly a similar scaling as in Eq. (17), but from a different point of view. They did not have to trace out the matter degrees of freedom explicitly. Instead, they argued that a black hole could be justly described by an ensemble of $N_{g} \gg 1$ gravitons. The authors further argued that the number of gravitons will suppress the effective interaction of a gravitons, i.e., given by $\alpha_{g} \leq 1 / N_{g}$. For a large $N_{g} \gg 1$, the black hole behaves like a coherent system, albeit a leaky one. The graviton escape signifies the Hawking evaporation of a black hole. Such a gravitational system with $N_{g} \gg 1$ can be perceived to be approaching a classicalisation limit, where extracting quantum features will be becoming extremely hard.

The other limiting case, when $N_{g} \ll 1$, provides us with an intriguing possibility of extracting the quantum nature of a graviton. This limit arises when $m \leq 10^{-5} \mathrm{~g}$, for such a system, the number of gravitons is less than one irrespective of the size of the box. Therefore, it seems that nature provides us with a window of opportunity where it might be possible to construct experiments cleverly to study the quantum nature of gravity for $m \leq 10^{-5} \mathrm{~g}$. Indeed, if $m \ll 10^{-5} \mathrm{~g}$, it will be again harder to probe or extract any quantum features. It has been experimentally possible to create macromolecules $m \sim 10^{-19} \mathrm{~g}$ over spatial superposition for $\sim 0.25 \mu \mathrm{m}$, or atoms $m \sim 10^{-21}$ g over $\left.\sim 0.5 \mathrm{~m}\right)$ [29, 30]. In all these cases, $N_{g} \sim 10^{-28}$, or $10^{-32}$, way too small to observe any detectable features of quantum gravity.

The situation appears to be quite familiar in cosmology as well if we were to assume that a world-line can describe the entire observable patch of the Universe. In that case, the mass contained in the Universe would be $m \sim \rho \times V$, where $\rho=H^{2}(t) M_{p}^{2}$ is the constant energy density of the Universe, and $V \sim H^{-3}(t)$ is the observable volume, which would scale with Hubble expansion rate $H(t)$ of the Universe. If we were now to compute the occupation number of gravitons within my observable patch of the Universe, we would find,

$$
N_{g} \sim\left(M_{p} / H(t)\right)^{2} .
$$

In the current Universe, $H \sim 10^{-42} \mathrm{GeV}$, which gives $N_{g} \sim 10^{121}$, which indicates that the space-time is perhaps very close to a classical description, and the number of gravitons again scales as the Area-law, $N_{g} \sim$ Area $/ 4 \mathrm{G}$, because the observable area of the Universe will scale as $\sim H^{-2}(t)$.

On the other hand, we already have a constraint on the largest scale in the Universe indirectly. Since we have not seen any primordial gravitational waves, or the B-mode polarisation in the cosmic microwave background radiation, we believe that the scale of cosmic inflation cannot be arbitrarily large. The latest Planck data places an upper bound on $H \sim H_{\text {inf }} \sim 10^{13} \mathrm{GeV}[12]$. Of course, we have invoked here primordial inflation as a mechanism to seed the fluctuations in the cosmic microwave background radiation. However, even for such a high energy probe, the number of gravitons present in the Hubble radius during inflation would behave nearly classically, i.e. $N_{g} \sim 10^{12}$, and $\Delta N_{g} / N_{g} \sim 10^{-6}$.

Finally, we can ask how robust our analysis is with regards to the nature of classical gravity. Note that we have not assumed any specific form of gravitational action. The only assumption we have made here is that the gravitons can be described by the massless degrees of freedom in a harmonic oscillator state with the minimal coupling to matter degrees of freedom given by $G \sim 1 / M_{p}^{2}$, which is valid for gravitational theories beyond 3 spatial dimensions. However, this argument will change if we insist on studying any higher derivative extension of gravity, which will bring inevitably a new scale in the problem, say $M_{s}<M_{p}$ in four space time dimensions, see [31, 32]. For such class of theories of gravity, we will have to tread the occupation number of gravitons carefully case by case.

Conclusion: We briefly conclude by highlighting that we have provided a rather model-independent constraint on the occupation number of gravitons in a quantum system determined by its mass and $M_{p}$ by tracing out the matter degrees of freedom. The occupation number is bounded by the Area-law, which is a reminiscence to Bekenstein's bound. Our bound suggests that the occupation number of gravitons in the black hole geometry will be bounded by the Area of a black hole, which is also the gravitational entropy of the object. For mass $m \geq 10^{-5} \mathrm{~g}$, the number of gravitons occupied within the gravitational radius, i.e. the Schwarzschild radius, is much larger than one. In an optimal bound on mass $m \sim 10^{-5} \mathrm{~g}$ would be ideal for extracting any quantum behaviour of a graviton. Furthermore, tracing out the matter degrees of freedom renders the space-time fairly classical within the observable patch of the Universe.

Acknowledgements: Authors would like to thank Roberto Casadio for helpful discussions. SB would like to acknowledge EPSRC grants No. EP/N031105/1 and $\mathrm{EP} / \mathrm{S} 000267 / 1$. AM's research is funded by the Netherlands Organisation for Science and Research (NWO) grant number 680-91-119. MT acknowledges funding by the Leverhulme Trust (RPG-2020-197). 
[1] C. Kiefer, "Quantum Gravity," "2nd ed", "Oxford University Press" (2007)

[2] S. N. Gupta, Phys. Rev. 96 (1954), 1683-1685

[3] G. Baym and T. Ozawa, Proc. Nat. Acad. Sci. 106 (2009), 3035-3040

[4] F. J. Dyson, ?The World on a String?, review of The Fabric of the Cosmos: Space, Time, and the Texture of Realitiy by Brian Greene, New York Review of Books, Volume 51, Number 8, May 13, (2004); T. Rothman and S. Boughn, Found. Phys. 36 (2006), 1801-1825

[5] Y. J. Ng and H. van Dam, Found. Phys. 30 (2000), 795805 W. A. Christiansen, Y. J. Ng and H. van Dam, Phys. Rev. Lett. 96 (2006), 051301

[6] G. Amelino-Camelia, J. R. Ellis, N. E. Mavromatos, D. V. Nanopoulos and S. Sarkar, Nature 393 (1998), 763765; G. Amelino-Camelia, Living Rev. Rel. 16 (2013), 5

[7] M. Parikh, F. Wilczek and G. Zahariade, Int. J. Mod. Phys. D 29 (2020) no.14, 2042001

[8] M. Toroš, T. W. van de Kamp, R. J. Marshman, M. S. Kim, A. Mazumdar and S. Bose, Phys. Rev. Research 3, 023178 (2021)

[9] S. Bose, A. Mazumdar, G. W. Morley, H. Ulbricht, M. Toroš, M. Paternostro, A. Geraci, P. Barker, M. S. Kim and G. Milburn, Phys. Rev. Lett. 119 (2017) no.24, 240401

[10] C. Marletto, V. Vedral, Phys. Rev,Lett. 119, 240402 (2017).

[11] R. J. Marshman, A. Mazumdar and S. Bose, Phys. Rev. A 101 (2020) no.5, 052110

[12] N. Aghanim et al. [Planck], Astron. Astrophys. 641 (2020), A6

[13] J. Martin and V. Vennin, Phys. Rev. D 96 (2017) no.6, 063501 doi:10.1103/PhysRevD.96.063501; J. Martin, Universe 5 (2019) no.4, 92

[14] A. Ashoorioon, P. S. Bhupal Dev and A. Mazumdar, Mod. Phys. Lett. A 29 (2014) no.30, 1450163

[15] J. D. Bekenstein, Phys. Rev. D 23 (1981), 287

[16] L. Bombelli, R. K. Koul, J. Lee and R. D. Sorkin, Phys. Rev. D 34 (1986), 373-383
[17] M. Srednicki,

[18] G. 't Hooft, Conf. Proc. C 930308 (1993), 284-296

[19] L. Susskind, J. Math. Phys. 36 (1995), 6377-6396

[20] M. Rakhmanov, Class. Quant. Grav. 31 (2014), 085006

[21] Weinberg, Steven. "Gravitation and cosmology: principles and applications of the general theory of relativity." (1972).

[22] M. Toroš, A. Mazumdar and S. Bose, [arXiv:2008.08609 [gr-qc]].

[23] C.C. Gerry, and P.L. Knight. Introductory quantum optics. Cambridge university press; 2005.

[24] G. Dvali and C. Gomez, Fortsch. Phys. 61 (2013), 742767; G. Dvali and C. Gomez, arXiv:1212.0765 [hepth]; G. Dvali, D. Flassig, C. Gomez, A. Pritzel and N. Win- tergerst, Phys. Rev. D 88, no. 12, 124041 (2013), [arXiv:1307.3458 [hep-th]]; G. Dvali and C. Gomez, Eur. Phys. J. C 74, 2752 (2014), [arXiv:1207.4059 [hepth]]; G. Dvali and C. Gomez, JCAP 1401, 023 (2014), [arXiv:1312.4795 [hep-th]].

[25] G. Dvali, C. Gomez, R. S. Isermann, D. Lüst and S. Stieberger, Nucl. Phys. B 893 (2015),

[26] G. Dvali, JHEP 03 (2021)

[27] R. Casadio, A. Giugno and A. Orlandi, Phys. Rev. D 91, no. 12, 124069 (2015), [arXiv:1504.05356 [gr-qc]]; R. Casadio, A. Giugno and A. Giusti, Phys. Lett. B 763, 337 (2016), [arXiv:1606.04744 [hep-th]]; R. Casadio, [arXiv:2103.14582 [gr-qc]].

[28] R. Ruffini and S. Bonazzola Phys. Rev. 187, 1767 (1969).

[29] T. Kovachy, P. Asenbaum, C. Overstreet, C.A. Donnelly, S.M. Dickerson, A. Sugarbaker, J.M. Hogan, M.A. Kasevich. Nature 528 (2015) no.7583, 530-533

[30] Y.Y. Fein, P. Geyer, P. Zwick, F. Kiałka, S. Pedalino, M. Mayor, S. Gerlich, M. Arndt, Nature Physics 15 (2019), 1242-1245

[31] T. Biswas, E. Gerwick, T. Koivisto and A. Mazumdar, Phys. Rev. Lett. 108 (2012); T. Biswas, A. Mazumdar and W. Siegel, JCAP 03 (2006),

[32] L. Buoninfante and A. Mazumdar, Phys. Rev. D 100 (2019) no.2, 024031 A. S. Koshelev and A. Mazumdar, Phys. Rev. D 96 (2017) no.8, 084069. 